\title{
Game play in vocational training and engineering education*
}

\author{
Bjarne A. Foss ${ }^{1}$ Ole K. Solbjørg ${ }^{2}$ Tor Ivar Eikaas ${ }^{3}$ Frank Jakobsen ${ }^{1}$ \\ ${ }^{1}$ Norwegian University of Science and Technology, Trondheim, Norway \\ ${ }^{2}$ NTNU Social Research Ltd., Trondheim, Norway \\ ${ }^{3}$ Cyberlab.Org AS, Trondheim, Norway
}

\begin{abstract}
Educational games may create a new and improved learning culture by drawing advantage of the new knowledge and skills of today's students obtained from extensive use of interactive game software. This paper presents a design basis and online learning resources taking advantage of game-related features like a high degree of interactivity, attractive graphics, a dynamical virtual universe, and an incentive system to promote prolonged and more advanced use. The educational resources, denoted PIDstop, are targeted towards the engineering domain. Feedback from over 2000 users clearly indicates that PIDstop has a positive learning effect. Training packages for vocational training of Automation Technicians is emphasized in this paper. Such learning resources must have a limited mathematical complexity; hence, the representation should be rather descriptive. Evaluation of learning resources to assess the actual learning effect is important, and a two-step procedure based on formative and summative evaluation is proposed for this purpose.
\end{abstract}

Keywords: Dynamic simulation, games, technical education, elearning.

\section{Introduction}

Technical education faces a number of challenges because of the recruitment situation in many countries within the engineering domain. According to the International Council of Academies of Engineering and Technological Sciences Inc attracting young students into careers as engineers, technologists, or technicians is a serious problem (CAETS, 2003). Another citing describes the situation in the U.S. who has lost $25 \%$ of its undergraduate population in engineering between 1982 and 2000 (Orsak, 2003). Today's students have knowledge and skills from extensive use of computer or video games, or more generally interactive game software (Prensky, 2001b). A computer game is in

\footnotetext{
*Published at 7th IFAC Symposium on Advances in Control Education, Madrid, Spain, June 21-23, 2006.
}

principle a game composed of a computer-controlled virtual universe that players may interact with in order to achieve a goal. Characteristics of such games are a high degree of interactivity, advanced graphics, a highly dynamical virtual universe, and an incentive system to promote prolonged and more advanced use. Prensky (2001b) provides an in-depth discussion of the fact that the students have radically changed in the sense that they think and process information differently from predecessors. Today's students have grown up with computers and computer games, and Prensky (2001b) refers to them as digital natives since they can be viewed as "native speakers" of the new technology. There is a growing understanding that educational games may create a new and improved learning culture (Prensky, 2001a). New models that integrate learning theory and educational games are emerging (Amory 
and Seagram, 2003), (Kiili, 2005b), and also learning resources based on such models are materialising (Kiili, 2005a). This paper is composed as follows: The use of simulations and games as a means to develop elearning resources is discussed in Section 2. Thereafter, in Section 3 and 4, the prototype system used herein is presented with emphasis on resources for vocational training purposes. Pedagogical evaluation is discussed in Section 5 before some conclusions end the paper.

\section{Simulations and games for learning purposes}

The development of ICT solutions has allowed for more complex and advanced representations of learning material. In this context, dynamic simulators can be regarded as a further development of visualisations, driven by mathematical models, and with a high degree of user interaction. The visual packaging of online learning resources is important and a parameter that influences the learning effect of an elearning resource. To exemplify, the visual image of an elearning resource is shown in Fig. 1. The graffiti-inspired image may seem strange to a mature audience. It has, however, been very well received by present undergraduate students as will be discussed later. A dynamic simulator reproduces, through the use of a mathematical model, the dynamic behaviour of a physical or abstract system such as the mass-damper-spring system in Fig. 1. Such simulators provide a two-sided advantage. First, they support the concept of a dynamic universe, and second they can be included in online resources applicable to the engineering education. This will become apparent during the next sections. When developing elearning resources, there are several aspects to take into consideration. One may start by defining what the learning outcome should be for students using the resource. Further, it is essential to note that one simulator can be used for learning at different levels. A simulator may be used for learning more fundamental aspects of a process, or alternatively the simulator may be used to present a concept without emphasis on the underlying theoretical aspects. It all comes down to packaging, ie. the form of representation and multiple views used. One may decide to present for instance a physical mechanism, like in the mass-damper-spring system in Fig. 1, in its most simple form. However, one may improve the students possibility for grasping the concept presented by relating the simulation to real life settings theoretically speaking, by relating it to the students existing knowledge, allowing them to reflect and build on this knowledge. There are a number of factors to consider when turning a dynamic simulator into a game that is motivating, and at the same time maintains pedagogical credibility and offer a realistic setting that supports the students process of reflection and knowledge development. Computer games are increasingly realistic, bordering on cinematic quality. Education institutions will never be able to compete with these games with respect to playability, or sophistication in graphics and audio. Still, it is possible to make stimulating and interesting games simulations in $2 \mathrm{D}$ with simple audio effects. Some important factors to develop successful simulation games are, as we see them:

- The metaphor or context chosen for the game should be easily recognizable.

- The role of the process being simulated must be obvious in the game.

- The game should comply with physical laws (which is not necessarily the case in traditional computer games).

- The goal of the game should not be fully achievable through trial and error.

- Sound effects are not vital, but could be incorporated to prevent the game from appearing too simplistic.

- The use of humour is expected to increase motivation for users at all educational levels.

- There should be several levels. This will in itself give the student valuable feedback on her or his progress and increase motivation.

- Rankings, using time or points as scores, gives a competitive edge that will increase the probability that students try several times.

\section{The PIDstop system}

The name of the prototype system used in this paper is PIDstop. The name arises from a merger of the terms "pitstop" and "PID-control". Pitstop is a place to fuel or more generally perform maintenance of a racing car, the analogy in our context is "tanking up knowledge". PID-control is an important term within the control engineering domain, the starting point of PIDstop in 2002. PIDstop can be integrated into any modern Learning Management System (LMS). The system is implemented using HTML/CSS, powered by a PHP server for dynamic content and mySQL database for storing user data. The dynamic simulations and games are implemented as Java Applets. This means that 


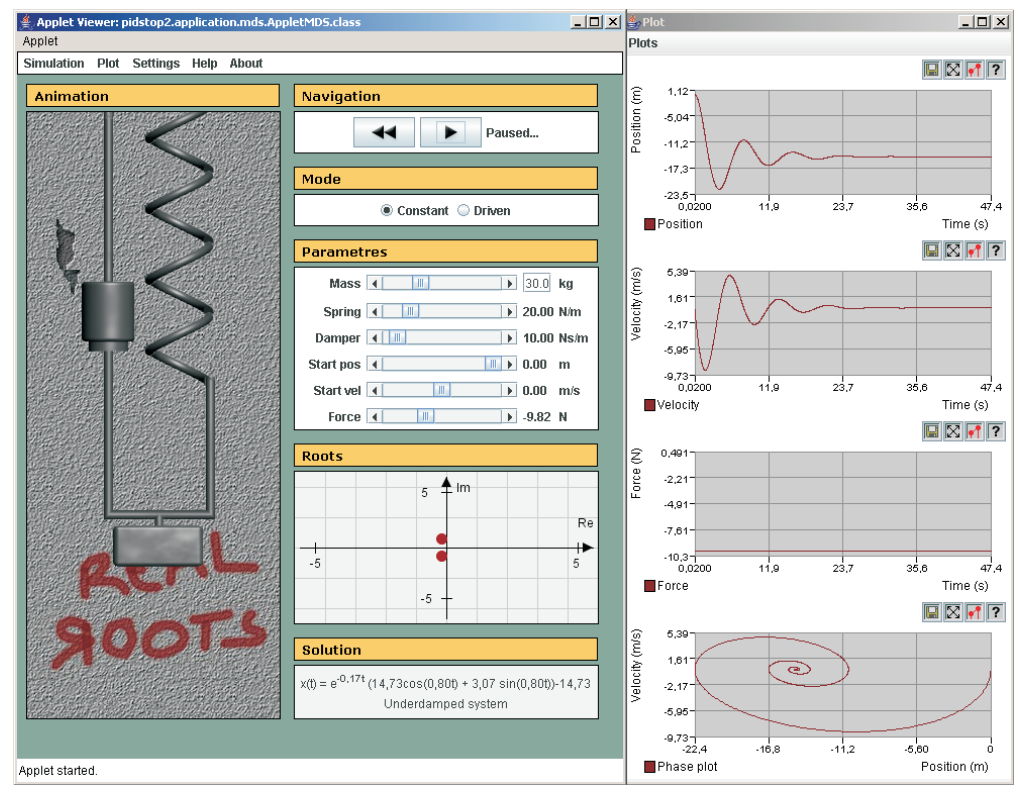

Figure 1: An elearning resource with multiple views of a mass-damper-spring system.

PIDstop learning resources are available to anyone with a Java-enabled browser. During the last couple of years a number of online learning resources have been developed within the PIDstop system, covering processes and systems like harmonic oscillators, magnetic levitation trains, autopilots, bungee jumps, DC motors, etc. (Foss and Eikaas, 2006), (Eikaas et al., 2006). An example using PIDstop-based simulators and games in nonlinear control education is given in Haugom et al. (2006). An example of a game-based elearning resource is shown in Fig. 2. It illustrates an inverted pendulum where the simulator is wrapped into a game where the user needs to balance the fuelling hose in an upright position and position the cart below the helicopter in order to refuel it. The game includes wellknown game features: The user has a number of "lives" available; there is a highscore list, animations, and multiple views. This game is part of an elearning resource which has been used in mathematics course at the Norwegian University of Science and Technology (NTNU). More than 2000 students have used PIDstop within the engineering education programmes at NTNU. This covers eight courses in mathematics, physics and control engineering. Formalized evaluation from this cohort has clearly shown that educational games provide a useful concept for online learning resources within the engineering domain, see Foss and Eikaas (2006) for more details. A PIDstop-based dynamic simulation game based on a magnetic levitation train was used for the webbased recruitment campaign for NTNU in 2005. During the three-month campaign, more than 8000 completed simulation-runs were recorded. It is not easy to measure user satisfaction of Internet-based campaigns like this, but the web-logs indicate that the average user played the simulation-based game approximately 5 times. A number of simulation-based games and competitions have been developed for industrial trade fairs and exhibitions in addition to a number of exhibitions focusing on technology dissemination towards young children (from approximately 10 years and up). The response has been very promising, even when using dynamic simulators adapted from advanced use at the university level.

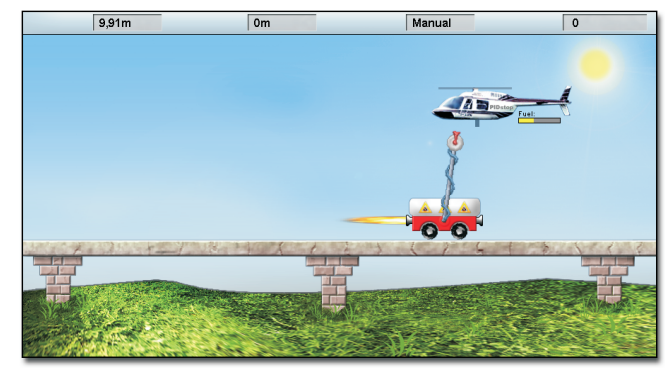

Figure 2: An inverted pendulum used in a game setting.

\section{Vocational training}

In this section we will provide some background on Automation Technicians, the target group for the AutoTech project. The AutoTech project is sponsored 
by the Leonardo da Vinci programme. The project includes partners from Romania, Germany, Spain, Switzerland and Norway, and its focus is on elearning for Automation Technicians. Selected elearning resources will be described as well as some initial feedback on these resources.

\subsection{Automation Technicians and training needs}

The main goal of the AutoTech project is to develop a set of new, motivating and innovative Internet based vocational training packages for Automation Technicians. The title used for this target group varies among the European countries (eg. Automatiker (NO), Automatiker/in (DE), Automaticien/ne (FR), Técnico en control de procesos (ES)). Typically, an Automation Technician has undergone a two to four years initial vocational training (upper secondary education), often combined with a period as an apprentice in a private enterprise. The daily work of an Automation Technician is quite technical, and requires practical experience and a good understanding of basic principles of automation and control systems in order to be able to maintain, trouble-shoot and solve problems related to automation and control of industrial processes. As a result of the developed learning resources, Automation Technicians will gain knowledge and practical experience in the design, tuning and troubleshooting of industrial control systems. This is an area where there is a documented significant potential for improvements in most industrial sectors. Badly or non-optimally tuned control loops are the origin of many operational problems like reduced product quality, increased production costs, environmental problems, etc. Automation Technicians play an important role in the day-to-day operation and maintenance of automation equipment and control systems in all process and manufacturing industries. The Automation Technicians do gain basic understanding and knowledge about automation and control systems from the initial vocational training in automation provided by an educational institution. In spite of this, there is a documented need for specialised, continuous training and practicing beyond the basic education. This is also due to organisational issues in many companies: The Automation Technicians are often given less opportunity for attending training courses etc. in order to update their skills compared to other groups with higher education. Even in large companies, there is often only a very small group of people having a background from automation and control. This limits the Automation Technicians opportunity to discuss problems related to their profession with in-house personnel.

\subsection{PIDstop learning resources}

The learning resources do have clearly defined learning objectives and focus on typical industrial control applications such level control, temperature control, and quality control for instance pH-control. Each application will encompass appropriate control structures. Such structures may include ratio control, cascaded control, override control, and split range control. Further, issues like control input saturation, nonlinear behaviour, measurement noise, bumpless transfer, and tuning are addressed. The training

package includes theory, quizzes, simulators, games, and an evaluation part. The theoretical part is descriptive with limited mathematical complexity. There are several quizzes for different knowledge levels. This provides the students with immediate feedback on their progress. Fig. 3 shows a screen dump from a typical user session. Emphasis is placed on flexibility, attractive graphics and multiple views. In this case simulations can be studied through the animations on the left hand side, and through time series plots to the right. The learning resource is flexible in the sense that may be run in alternative modes manual mode, PID model, cascaded control mode and with a gain scheduling controller. In the latter case the graphics are changed by visualizing a tank with a varying crosssectional area. The user will have access to background material like PID-controller tuning rules. Further, a user can download the data from the test he/she has run so as document trials in a report. Preliminary feedback on this learning resource is that it is suitable for the purpose outlined. pH-control is the focus for the simulator shown in Fig. 4. It is shown in a more advanced mode than the learning resource in Fig. 3. It includes both ratio control as well as cascaded loops. Time series plots are not included in this figure. A useful feature of the learning resource is the ability to examine the interior of the PID-controller as shown in Fig. 5.

\section{Evaluation}

As mentioned feedback from a large cohort indicate that the there is benefit of applying the PIDstop concept in education, see Foss and Eikaas (2006). The evaluation procedure in the AutoTech project has been developed in close cooperation with pedagogical competence. This procedure will be presented and discussed in the sequel. The potential for improved motivation and learning quality through the use of simulation based gameplay has been substantiated through earlier evaluation. Still, though the subject matter may be clearly defined and the simulations are accu- 


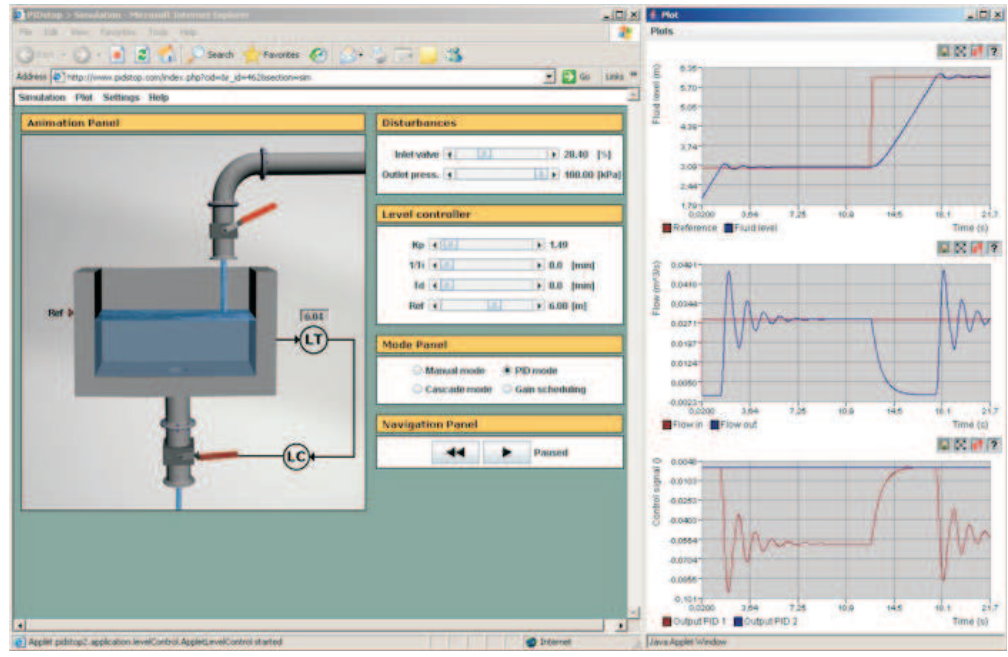

Figure 3: A level control learing resource for Automation Technicians.

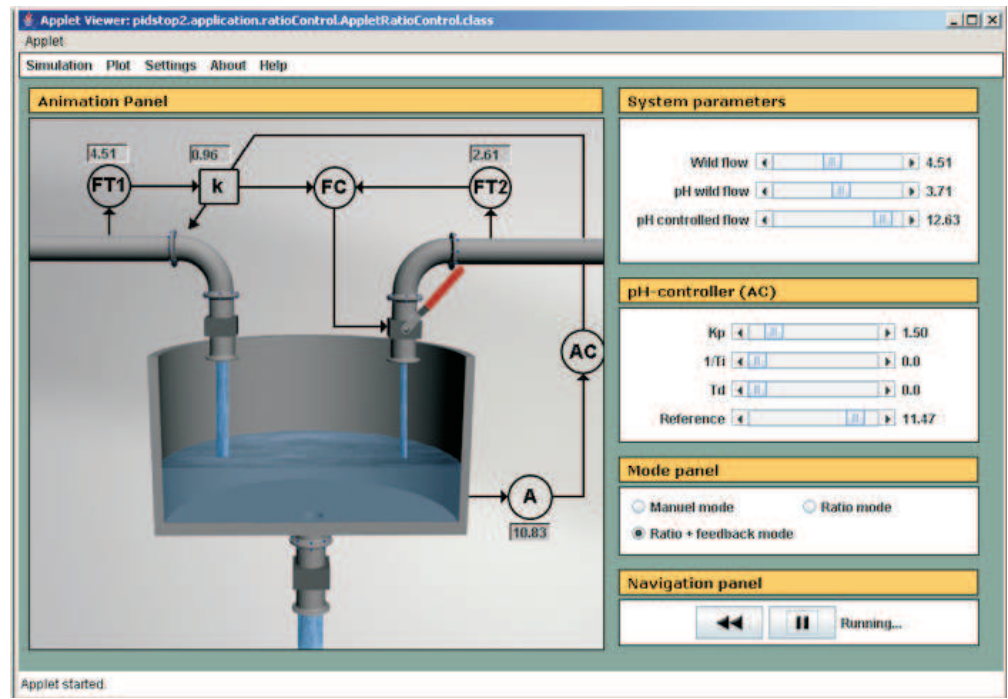

Figure 4: pH control learning resource for Automation Technicians.

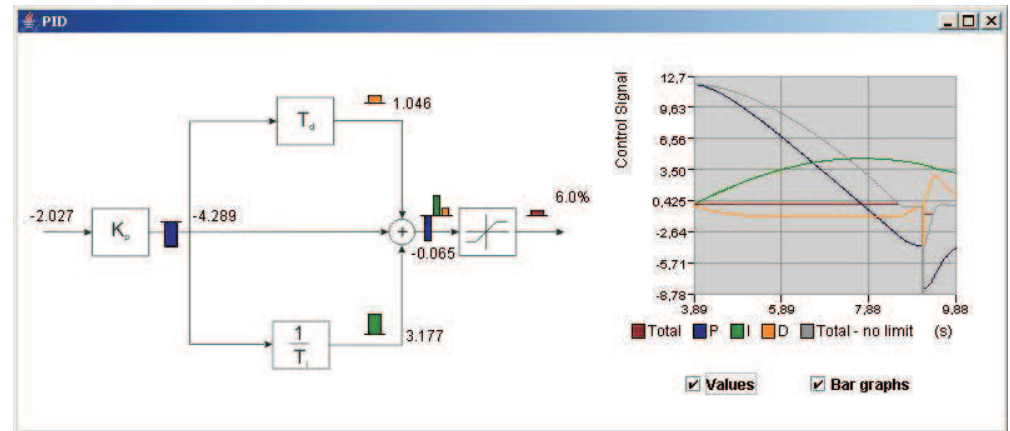

Figure 5: Display of the interior of the PID-controller. 
rate, an improved outcome of the learning process is not granted. There are many aspects important for the quality of the resources and the quality of the learning experience itself. Though simulations and games are designed to reduce complexity and enhance learning, the development process of a learning resource itself is quite complex. To ensure quality throughout the development process and also monitor the perceived outcome of using the resources, a thorough evaluation will be performed involving all partners. In addition to important aspects mentioned earlier, such as usability and motivation, the AutoTech project also has users with varying background. Although the main target group is Automation Technicians, also students in related subjects will be targeted. The countries involved are different with regards to the training needs related to industrial focus, higher education curricula, skills and experience in using ICT; all these and many more factors may influence the efficiency of the games and simulations as learning resources. As evaluation has to take all this into account, the evaluation must therefore also be conducted as efficiently as possible to reduce strain on the partners, which should be allowed to allocate their resources to development. The choice has been made to perform evaluation in two steps; one during the development process, and one after the resources have been applied for in-service training of $\mathrm{Au}-$ tomation Technicians. This may be seen as a combination of formative and summative evaluation. Formative evaluation is conducted during a process, often involving many stakeholders, to improve both the following process and the final outcome. Summative evaluation is conducted at the end of the process to establish whether processes and products fulfill the set criteria (Scriven, 1991). It should be noted that evaluation in this project focuses on the developed learning resources, their fitness of purpose, and their learning outcome. This allows also for the formative evaluation to give constructive input to the development process, and thus to increase the quality of the final resources. For evaluation of learning resources and their learning outcome, a number of methods are readily available, such as interviews, observations, user logs and usability tests (Reeves and Hedberg, 2003). Within the scope of the evaluation part of the AutoTech project, questionnaires are chosen as the main method for evaluation. It is efficient, targeted, and reduces the problems that differing languages and cultures between partners infer. To accommodate for evaluating the resources with regards to both students as a secondary group and Automation Technicians as the primary group, students in relevant educational settings are used as a test panel during the formative evaluation stage. This gives targeted feedback on the quality of the learning resources, as well as the perceived learning outcome. Students background and goals may of course differ from those of the Automation Technicians, but this is actually an advantage as the learning objects should be adaptable with regards to the academic level and learning goals. During the second evaluation stage, the resources are used for in-service training of Automation Technicians, which establish the relevance, perceived quality of the resources and learning outcome for the primary target group. This group may also be followed up at a later point to answer whether some other indicated goals of the project have been fulfilled, such as increased job satisfaction and increased job performance. For further development of learning packages, the evaluations will also give input as to which parts of training packages are perceived as most valuable for learning, and thus should be emphasized.

\section{Conclusions}

This paper presents a concept for developing elearning resources based on dynamic simulators and features from computer games. This concept has many appealing features to the generation presently starting or following engineering programs in higher education or vocational training programs in high schools. Tests, using the PIDstop system, on a large cohort have verified this. Training packages for vocational training differ from engineering education. In particular the level of mathematical complexity must be reduced. Hence, the representation should be more descriptive. Evaluation of learning resources to assess the actual learning effect is important. A two-step procedure based on formative and summative evaluation is proposed for this purpose.

\section{Acknowledgments}

The authors gratefully acknowledge the support from the European Commission through the Leonardo da Vinci programme, contract N/04/B/PP 165.011 AutoTech, the VOX project Remotely laboratory process in continued education for Automation Technicians, The Norwegian University of Science and Technology (NTNU) and Cyberlab.Org AS.

\section{References}

Amory, A. and Seagram, R. Educational game models: Conceptualization and evaluation. South African Journal of Higher Education, 2003. 17:206-217. 
CAETS. Future engineering challenges. 2003. URL http://www.caets.org/nae/naecaets.nsf/ weblinks/NAEW-5CAJK5?OpenDocument.

Eikaas, T. I., Foss, B. A., Solbjørg, O. K., and Bjølseth, T. Game-based dynamic simulations supporting technical education and training. International Journal of Online Engineering, 2006. 2(2).

Foss, B. A. and Eikaas, T. I. Game play in engineering education - concept and experimental results. International Journal of Engineering Education, 2006. $22(5)$.

Haugom, R., Solbjørg, O. K., Pettersen, K. Y., and Eikaas, T. I. Simulation game in nonlinear control theory education. In Advances in Control Education. Madrid, Spain, 2006 .

Kiili, K. Content creation challenges and flow experience in educational games: The it-emperor case. The Internet and Higher education, 2005a. (8):183-198.

Kiili, K. Digital game-based learning: Towards an experimental gaming model. The Internet and Higher education, 2005b. (8):13-24.

Orsak, G. C. Guest editorial k-12: Engineering's new frontier. IEEE Trans. Education, 2003. 46:209-210.

Prensky, M. Digital game-based learning. McGraw-Hill, 2001a.

Prensky, M. On the horizon. NCB University Press, 2001b. 